International Conference on New Interfaces for Musical Expression

\title{
Discourse is critical: \\ Towards a collaborative \\ NIME history
}

\section{S. M. Astrid Bin ${ }^{1}$}

\author{
${ }^{1}$ Ableton AG \\ License: Creative Commons Attribution 4.0 International License (CC-BY 4.0).
}




\section{Abstract}

Recent work in NIME has questioned the political and social implications of work in this field, and has called for direct action on problems in the areas of diversity, representation and political engagement. Though there is motivation to address these problems, there is an open question of how to meaningfully do so. This paper proposes that NIME's historical record is the best tool for understanding our own output but this record is incomplete, and makes the case for collective action to improve how we document our work.

I begin by contrasting NIME's output with its discourse, and explore the nature of this discourse through NIME history and examine our inherited epistemological complexity. I assert that, if left unexamined, this complexity can undermine our community values of diversity and inclusion. I argue that meaningfully addressing current problems demands critical reflection on our work, and explore how NIME's historical record is currently used as a means of doing so. I then review what NIME's historical record contains (and what it does not), and evaluate its fitness for use as a tool of inquiry. Finally I make the case for collective action to establish better documentation practices, and suggest features that may be helpful for the process as well as the result.

\section{Introduction}

NIME's most defining characteristic is its interdisciplinarity. We are a "heterogenous field of experimentation where many ideas and design strategies exist in parallel"[1]]. This deep interdisciplinarity is one of NIME's greatest strengths, but also presents one of our most enduring challenges. As NIME has stretched to incorporate a huge range of approaches and techniques from other disciplines, we have also inherited varying notions of what constitutes knowledge that is valuable and important.

There is recent evidence that these latent influences are having negative effects. For example, NIME's authorship has little gender diversity[2]], our community has no explicit political position which risks implicitly supporting the status quo[므], practicebased research has long struggled to find a place of legitimacy in NIME[4]]. Hayes and Marquez-Borbon[트] call this an "epistemological crisis", and called on NIME to proactively address it. This paper departs from that point: How to address it.

I begin by exploring the features of NIME discourse. I briefly trace the history of NIME's adoption of outside perspectives and techniques. I then describe the value of 
this interdisciplinarity as well how it has introduced values that are sometimes in opposition, thereby unpacking evidence of these latent tensions causing these recentlyhighlighted problems. I assert that, to meaningfully address these tensions and conflicts, we will have to understand the interplay of our various included disciplines.

Next, I describe NIME's historical record. I describe ways it is already being employed as a tool of critical reflection, and why this record is perhaps the best tool we have to understand our work. I explain how a more complete record can help us make explicit the forces that impact work that is considered important - forces that may work against our own values. I discuss the current recorded NIME history, and critique its ability to reflect the breadth of this community's work.

Finally, I make the case for collective action to address these urgent issues. I describe how a better record would allow us insight on our work and its development. I also examine how the larger process of discussing this record - what it should include, how it could be used and by whom - would be an important process of critical discourse in itself. Finally, I describe some features of this process that might be helpful, possible extensions to this work, and the role they have to play in the way we think about ourselves, our work and each other.

\section{NIME and its discourse}

First, a distinction. In this paper I refer to the catalogued archive of outputs, such as publications, performances and installations as NIME's "historical record".

Additionally, I want to draw a vital distinction between this catalogued output and the wider NIME discourse. While catalogued outputs form the historical record, discourse is a continuous process of exchange which includes every other facet of NIME activity: the instruments we make, the conversations we have, the relationships we maintain, trends that emerge, and so on. Discourse is not equivalent to published output; rather, the historical record is a product of our discourse. Discourse contains dominant ideas as well as those challenging the status quo[ㅁ], structures of power, conscious and unconscious ideas, as well as emotional dynamics[7]. Most importantly, it is through discourse that we develop ways of judging which work is important and valuable, and as a result the work that makes up our peer-reviewed historical record.

\section{Interdisciplinarity and complex discourses}

While an in-depth discussion of interdisciplinarity is outside the scope of this paper, it is useful to clarify this term in this context. Disciplines are areas of study that have, to 
varying degrees, well established ontologies and conventions of arriving at and recognising knowledge. Though sometimes rigid, disciplinary boundaries can be valuable because it allows a discipline to be "able to account for its conditions of existence and thus ... how it arrives at knowledge and practices".[ㅁ]

This clarity of knowledge and practice gets hazy when disciplines merge. Born and Barry state that interdisciplinary activity is "an attempt to integrate or synthesise perspectives from several disciplines"[]], and qualify this by specifying that though it indicates combined disciplines, interdisciplinarity should not be understood as the sum of the contributing disciplines as the boundaries between them "are neither entirely fixed nor fluid; rather they are relational"[]]. Though this interplay can result in exciting new approaches and perspectives, it also brings with it complexity that leads to "lack of available criteria to assess interdisciplinary work on its own terms"[10].

\section{The evolution of this community}

Though the practice of exploring how to use technology to make musical instruments stretches back far further, New Interfaces for Musical Expression emerged as a community concerned with digital musical instruments (DMIs, or interfaces that control a sound-generating computer[11]) through a workshop at the SIGCHI conference in 2001. Our origins in the human-computer interaction (HCI) community mean that we adopted a range of values and approaches from HCI that have deeply influenced our approach to making DMIs (as evidenced through our enduring interest in "evaluating"[12] instruments, the approach to musician as "user"[13], adopted design practices[14], and so on). Along with HCI perspectives the study of DMIs has also readily included perspectives from various branches of computer science as we leverage ever-smaller, ever-cheaper and and ever-more-powerful computers, from sound synthesis, digital signal processing, electronic engineering, innovative sensor techniques, robotics, and machine learning.

The complexity does not stop there. Applications of DMIs in performing arts[트] ,

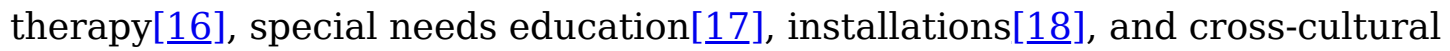
collaboration[19] show the breadth of impact of our work, and explodes DMI use cases into an array of domains. As DMIs often require constructing a physical object, we have drawn on both modern digital techniques[20][21] as well as traditional instruments[22][23] for inspiration and insight.

This does not trace all of NIME's influences, but demonstrates that new techniques, perspectives and disciplines are readily integrated into this community: "The walls are 
elastic, everyone fits in"[24]. This flexibility means that this community is a place where researchers can combine and re-combine perspectives without typical disciplinary constraint, with unusual and insightful results[25][르][27].

What is conspicuous by its absence is work that reflects on the tensions inherent in this interdisciplinarity. Until very recently, works that contend directly with its implications have been thin on the ground, and virtually none that acknowledge NIME's own epistemological status quo, let alone levy a direct, explicit challenge to it. This emergence of recent critiques may be a sign of NIME's disciplinary maturity (we are, after all, in the 21st edition), but these recent criticisms should make us pause to consider the effects of simply incorporating new perspectives without critical reflection, and if this is sustainable. Strathern makes a salient point: Successful interdisciplinarity does not just mean seeking out new sources of synergy; instead, growth comes through productive forms of agreement and disagreement, and by finding "a successful way of re-engineering and distributing knowledge to break through old barriers and reach a new plateau of knowledge sharing"[28].

\section{The politics of knowledge}

NIME's deep interdisciplinarity means that there are wide breadth of perspectives on what important or valuable work is. These perspectives play out most directly through the reviewing process, as everything at NIME, from performances to workshops to papers, is peer-reviewed. This means that the varying perspectives in our discourses directly produce the output that becomes our historical record.

Appearing in the historical record is not free of political content. Publishing grants legitimacy, which is significant; for instance, legitimacy brings institutional support and funding, not to mention career development, and it signals that an avenue of inquiry is worthwhile. Hayes and Marquez-Borbon identify "the market demands of the neoliberal university"[్] as one of the underlying forces expressed here. This lens that may explain why, for example, practice-based research has struggled to establish itself as a legitimate approach despite it being a familiar way of working to so many: its outputs are valuable, but not easily quantified.

There are further, more latent effects. NIME's lack of critical reflection on our outputs means that we have not developed a variety of perspectives of on the historical importance or influence of existing work. For example, the editors of A NIME Reader[29], when having to choose 30 works for their collection, used Google Scholar citation counts as a metric for determining influence[뭉]. Though this was a well- 
intentioned method to maintain neutrality, it does not have neutral effects - for instance, as well as already being underrepresented in the NIME proceedings, relying on citations as a metric for determining importance disadvantages women. Evidence suggests that work by men is cited more often across a range of fields[풀, resulting in work by women being inherently perceived as less important. In this way, by attempting to remove human bias from the selection process by relying on external metrics to determine influence, the problem of underrepresentation of women in NIME that already exists is only further reinforced.

Though diversity has many axes, issues around gender diversity was the first dimension to gain focused attention within NIME as part of a recently-emergent trend of considering our wider social impacts and responsibilities $\underline{123}$. Xambo[2] reviewed the NIME publication archive from 2001-2017 and found extremely low numbers of women authors (14\%), with even fewer presenting work more than once. (It's notable that identifying women was done "manually", inferring gender through first names; anything other than a binary classification would be therefore impossible.) As a solution Xambo presents a case study of WiMT, a group at Georgia Tech aiming to "raise awareness" about underrepresentation of women in music technology, but does not offer insight on the causes of this problem, or offer suggestions to meaningfully address them.

There are a number of possible contributing causes of NIME's gender diversity problem, but without a critical analysis of NIME itself the causes are not clear and meaningful action is not possible. For example, the gender diversity problem in STEM is well established, is this the effect we're seeing? Could we be seeing a result of NIME privileging scientific work in recent years[4] , therefore reinforcing the value of the work from circles that exclude women? Are women as under-represented in performance, instrument making, workshops, and installations as they are in publications (the works that we have determined to be important enough to be documented)? Who is mentoring the women who are present? Challenges to the status quo require "cogent and compelling intellectual and creative justifications for a redistribution of attention to new objects of study, new perspectives on old disciplinary objects, and new conceptual and methodological resources"[32], and without being able to locate the cause or even reliably theorise on it, we are unlikely to be able to identify the forces undermining our social values, let alone take meaningful action to confront them. 


\section{NIME's historical record}

The main challenge in understanding our identity is that NIME (and, perhaps, music technology more widely) does not have a cohesive epistemological identity, so we do not have the benefit of established rules of a dominant discipline, or any prevailing ontology. To be clear, a lack of cohesive ontology is not a bad thing - NIME's elastic nature makes it the uniquely fertile ground that it is. Nevertheless this does pose a challenge, because when we try to understand our work we lack a readily-available disciplinary framing and find ourselves scrambling for tools. So far, the best one we've found is our own historical record.

\section{Historical record as tool of inquiry}

The challenges of NIME's interdisciplinary complexity have not gone unnoticed, and pondering the very nature of the knowledge created by this community and its identity is an enduring area of interest. NIME proceedings are peppered with reflections on

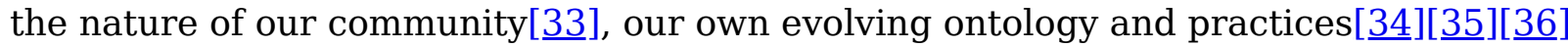

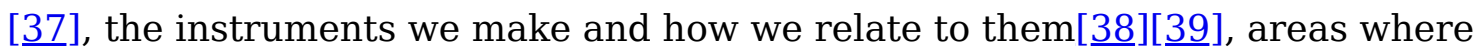

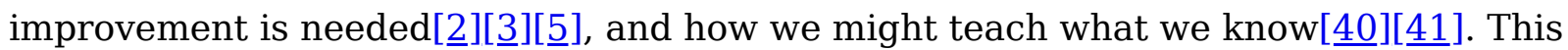
may be because, as we lack any dominant discipline, the community's output is the most stable epistemological ground we can stand on. Of course, examining the output itself is rarely the point (except when trying to illustrate something specific about our publications[2] ); instead, these reviews of the historical record look to gain insight on the nature of our work and the rules that produce it, and the character and identity of the discourse.

This use of the historical record highlights the importance of the records we create, and also suggests the vital importance of the quality of those records. However, critical reflection on what this archive does (and doesn't) represent, and by extension the insights analysis can offer, is conspicuously absent (though there is some acknowledgement that this record is incomplete[ㅌ6]). Because of its role as an instrument of inquiry, this is more than just a record - it's an important tool we use to understand our own work, perhaps the best tool available to us. As such, we must critically assess its fitness for this purpose, and improve its suitability for this task.

\section{What's in NIME's historical record}

The NIME community has dedicated significant efforts to centralising, cataloguing and preserving the published conference proceedings on the NIME website $\underline{4}$. These proceedings are also available as publicly-available Bibtex files on Github $\underline{5}$. In this 
Github repository are also records of musical performances and installations that have been presented at previous NIME conferences, but records only exist from 2008 (and even then not every year is represented). Further, no records of NIME workshops exist; the first day of NIME is typically dedicated to these peer-reviewed events, but a record of workshops is absent, let alone what happened at those workshops.

Perhaps most confounding is that there is no record of instruments. Papers that have attempted to understand instruments at NIME have relied on the proceedings to cobble a list of instruments that have appeared at NIME[42], but instruments that, for example, appeared in performances and for which there were no accompanying papers have no trace anywhere. On one hand perhaps this is understandable; it's much easier to archive, store and cite publications than it is to develop a way of documenting instruments, which include hardware, software, design files, instructions, and so on, and are largely idiosyncratic in nature. However, as we are a community with these artefacts at the core of our research, this lack of record - and even more the lack of urgency around it - is bewildering. (Interestingly, a workshop in 2016[43] pondered what a documentation system for NIME instruments might look like, but, perhaps ironically, no record of the workshop or its outcomes exists.)

NIME's historical record only really reflects published proceedings. While it is exhaustive and useful in this dimension, our work is much more. While demos and posters have associated papers, they are typically for hands-on instruments and tools, and a paper may not adequately capture their important contributions. Without a record of instruments it's impossible to know how these have been affected by trends written about in papers, or vice versa; we also can't trace the influence of instrument builders on each other, or look for their influence on artistic output. Without performances or installations we have no record of NIME concepts in practice, or how they reflected or were impacted by technological developments.

Turning outwards, we can do little more than speculate at how NIME work has affected (and been affected by) commercial music making, popular culture, trends such as the rise of the maker movement, sensor innovations, and so on. We also have no documentation of the people who make up this community except the names of authors. Since so much is missing from the best tool we have to understand our own work, our ability to critically engage with what we do, connect it to the outside world, see competing trends and tensions within it, or meaningfully identify and address problems of our own status quo is severely limited. 


\section{The documentarians are not coming}

NIME's historical record is incomplete, but the prospect of doing this work is overwhelming. We might well wonder: Aren't there documentarians who can (or should) do this work?

It is not the job of the NIME documentation team. Currently, the documentation of NIME proceedings relies on a small group volunteers $\underline{6} 7$, and developing new methods of documenting our history is a task far too large to be within their remit. Additionally, there's no critical community embedded enough in this work to take it on: Unlike many artistic areas of activity we have no community of objective critics who analyse NIME knowledge, processes and activity through a variety of lenses grounded in various cultures and histories. Further, no sub-group of NIME practitioners has emerged to take on the task of creating a critical NIME history that engages with the complex interplay of the contributing disciplines, or how NIME work relates to culture at large. (The beginnings of this kind of work exist[44] but are preliminary at best; this may be just as well, because establishing canon, or a single notion of what history is or what it means, would divest us of engagement with these issues and transfer that responsibility to a small group, when what we need is community-wide critical engagement.) Most importantly, documentation is is not a one-time job. A historical record is a living document that expands every year, so even if done once someone will need to maintain it.

In short: This task is urgent, massive, and belongs to all of us. Hayes and MarquezBorbon, when challenging us to unpick these conceptual knots, acknowledge that "the incentive for doing so may be counter-intuitive given the amount of care and labour that will be required"[]․ Collective community action is not only the way this work should be done, but also perhaps the only way it can be done.

\section{What we stand to gain}

There are a number of ways that richer data can lead to new and important insights. For example, technical details of instruments (such as the programming language used, the hardware platform employed, materials used) can provide valuable historical context, and, for instance, could allow us to trace how programming languages, hardware platforms, sensors, and so on affect our work, and even show us how our work is responding (or failing to respond) to social issues such as environmental concerns. Further, richer data could tell us about the people doing NIME work; currently there's no opportunity for authors to offer demographic information to form 
an anonymised aggregate representation of the authors and presenters at NIME each year, and as such there is no record of them other than name and affiliation at the time of publication. Along with better representation of gender expression, factors such as race, language, country of origin, and main discipline are just a few criteria that could give a better sense of the people present, as well as those not present.

More than simply being an objective pool of data, the value of a historical record is its process. Assessing the current historical record and engaging in a collaborative task of planning, collating and maintaining it signals this community's intentions. We have seen similar effects already: Since NIME has begun to directly engage with issues of diversity, disability and representation, these topics have become more prominent and found a place in our discourse. In the same way, we need to invite and create space for critical reflections on our past to gain an understanding of the latent assumptions, conventions, and dominant perspectives that affect us.

These debates exist in other fields. Vigorous critical challenges to the status quo has emerged in recent years in HCI along the lines of class[45], sexual orientation[46],

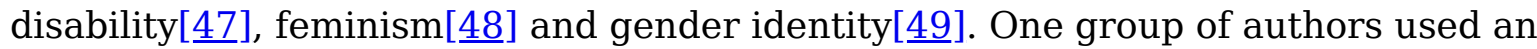
extended abstract to accuse CHI, the annual conference, of being "a tool that serves to reinforce the political and ideological status quo"[미] with the abstract consisting mostly of guitar tab and lyrics for an anti-HCI punk song. Levying that critique (at the very conference publishing the paper no less) is certainly an act intended to provoke, but it is precisely these types of provocations that provide the opportunity for critical discourse. Morreale et al[ㅍ] note that NIME needs these debates across lines of disciplines, identity, politics, culture, which are currently absent. Understanding our work requires a more complete historical record, but the most impactful thing may be the process of constructing it.

\section{Towards a collaborative historical record}

This is a community task, and it's impossible to be helpfully prescriptive about what this record should be or how this work should be carried out - that is important collective work. What I can offer are suggestions for features of both the process and the outcome that may help us usefully reflect on the existing record, and can serve as a starting point for discussion and planning.

\section{Feature 1: Collaborative}

This is not somebody's record; this is everybody's record. Though we will doubtless need a team willing to shepherd this process, the task of constructing an archive that 
reflects our work belongs to all of us, and wide perspectives are needed - those with good ideas about how to archive instruments may not be the people with good ideas about how to archive performances. Though focused workshops and discussion will be useful, the recent development of the NIME forum $\underline{8}$ means that planning can include far more voices.

\section{Feature 2: Ongoing}

This isn't a job for one year, it's a job for all years. This means that replicability is crucial, so yearly steering committees don't have to do the work of deciding what data to collect, and how. Replicability of process will mean that records are consistent, and that inevitable changes to what data is collected or how can be implemented later on.

\section{Feature 3: Flexible}

Without a doubt work within NIME will continue to expand and change, and what we document today may not reflect the work of the future. This process must be flexible enough to meet the needs of documenting outputs as they change and evolve.

\section{Feature 4: Openness}

Any documentation of our work should be openly available, and open to additions. In this we should consider not only where this data can be accessed, but how suggestions, submissions and corrections can be made. Github or other versioning platforms are ideal for this, especially with the in-built system of pull requests for submitting or correcting data and issue tracking for making suggestions, but as NIME increasingly includes practitioners outside of computer science we should consider how to document this process to make it more accessible to those who don't usually use versioning software.

\section{Feature 5: As complete as we can make it}

The NIME historical record will never complete, but we must continuously ask if this record accurately reflects this community's outputs (and include many perspectives in those conversations). There will doubtless be difficulties - for example copyright has been a stumbling block for recording performances in the past - but these challenges must not cause us to stop; an imperfect record is always preferable to a record that doesn't exist.

On the subject of recordings, though these are important documentation it's also important to have documentation that is searchable. Standardised templates for 
musical performances, installations and instruments could be helpful (this existed at NIME in $2019 \underline{9}$ but the information included should be reviewed and this practice made consistent).

\section{Reflecting the people doing the work}

Though careful consideration will have to be given to how to collect and store this data, giving NIME participants the optional opportunity to submit demographic data, anonymised and stored in aggregate, can help us understand the people doing this work, and how their perspectives, disciplines, social experiences, cultures, languages and musical traditions contribute to it. If we want to understand the root of our problem of a lack of representation of non-white, non-male, non-EuroAmerican work in this community we must be able to clearly see this problem, track it over time, and strive to understand factors that impact it.

\section{What about the past?}

The fact remains that NIME already has a two-decade history, and large amounts of it are unrecorded. Though establishing documentary practices for now and the future is the most urgent task, a documented and open method of contribution to the archive could allow those who came before to contribute their work. Not only does this place value on past work, but it also helps us improve and add dimension to the existing record. We also must keep in mind ways to bring the earlier record into parity; for example if we move away from Bibtex format for the future, we should create a copy of existing record to this new format as well.

\section{Where we might go in the future}

An important and challenging part of this process will be anticipating the forms this data should take. Currently all NIME records are in Bibtex format, which is useful for paper writing but may limit their usefulness in other applications. For example, knowledge graphs have long existed in the humanities to identify connections, trends, and lines of influence in complex archives[21], and technologies like graph databases[를 have made it possible to explore and manipulate huge datasets to gain new insights, and if this is a direction of interest formats like CSV are more useful. The field of digital humanities has much insight to offer here[ㅌ3], and it's worth taking these existing techniques into consideration when deciding on the form this data will take. 
Ultimately, we must keep in mind that, though the NIME historical record is a vitally important in fostering better critical discourse, it's not a panacea; rather, it's a first important step. But, the process of discussing the historical record can - and should - lead to essential wider discussions on how we understand our work and relate to the wider world, and how the tensions that underpin our discourse play out through other factors (such as peer review processes, nomenclature, and so on).

\section{Conclusion}

This year, NIME is 20. Community values are starting to emerge, and we're also starting to recognise that latent forces are acting on our work in ways that contradict those values. We are beginning to seek ways to meaningfully address them, but being able to fully understand the work we do and the people who do it - factors essential for levying critique - remain undocumented, as the NIME historical record has so far not captured them.

In this paper I traced the history of NIME's deep interdisciplinarity, and demonstrated how, with a lack of stable epistemological or ontological ground to stand on, we turn to our own output to understand the competing forces that underpin our work. This record is currently not representative of our work or community, and urgent collective action is needed to create one that is if we want to identify and address latent tensions that affect how we view, judge and understand our work. I offered suggestions of features for this process and its outcomes, as well as perspectives on the past and possible future.

Epistemological complexity is not a "problem" to be solved; it is NIME's willingness and ability to incorporate perspectives that makes this community as vibrant and innovative as it is. Rather, our challenge is that we have limited methods to understand how this complexity plays out in our work, and how to address forces that undermine our values. By addressing and understanding the interplay of disciplinary influences in the work that we do, we can not only produce work that reaches further and breaks more ground than ever before, but also model how other communities might act collaboratively, collectively, and meaningfully to understand the epistemological complexity of our contemporary world.

\section{Acknowledgements}

Thank you to my colleagues that provided invaluable input into this work: Fabio Morreale, Laurel Pardue, Andrew McPherson, Chris Peck, David Pocknee, and Braxton Sherouse. 


\section{Footnotes}

1. https://www.nime.org/diversity/ $\leftrightharpoons$

2. https://www.nime.org/environment/

3. https://www.nime.org/ethics/

4. https://nime.org/archives/

5. https://github.com/NIME-conference/NIME-bibliography $\triangleq$

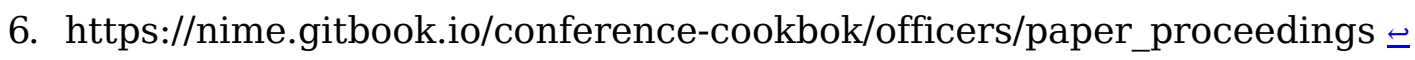

7. https://nime.gitbook.io/conference-cookbok/officers/music_proceedings $\subseteq$

8. https://forum.nime.org $\subseteq$

9. https://nime.gitbook.io/conference-cookbok/officers/music_proceedings $\subseteq$

\section{Citations}

1. Elblaus, L., Hansen, K., and Bresin, R. 2014. NiME design and contemporary music practice: Benefits and challenges. Workshop at New Interfaces for Musical Expression. $\subseteq$

2. XАмBÓ, A. 2018. Who Are the Women Authors in NIME? Improving Gender Balance in NIME Research. .

3. Morreale, F., Bin, A., McPherson, A., Stapleton, P., and Wanderley, M. 2020. A

NIME of the Times: Developing an Outward-Looking Political Agenda For This

Community. New Interfaces for Musical Expression. $\_$

4. GuREvich, M. 2016. Diversity in NIME research practices. Leonardo 49, 1, 80-81.

5. Hayes, L. and Marquez-Borbon, A. 2020. Nuanced and Interrelated Mediations and Exigencies (NIME): Addressing the Prevailing Political and Epistemological Crises.

New Interfaces for Musical Expression. $\subseteq$

6. Diamond, I. AND QuinBy, L. 1988. Feminism and Foucault: Reflections on Resistance. $\cdot \subseteq$

7. WeEdon, C. 1987. Post-structuralist theory and feminist practice. Oxford:

Blackwell. $\_$ 
8. Strathern, M. 2004. In crisis mode: A comment on interculturality. Commons and Borderlands Working Papers on Interdisciplinarity, Accountability and the Flow of Knowledge. $\triangleq$

9. BARRY, A. AND BoRN, G. 2013. Interdisciplinarity: reconfigurations of the social and natural sciences. Routledge. $\bullet$

10. Strathern, M. 2005. Experiments in Interdisciplinarity. Social Anthropology 13, 1, 75-90. Quoting online discussion $ヒ$

11. VAn Nort, D. And CASTAgné, N. 2007. Mapping in digital musical instruments. In: Enaction and enactive interfaces: A handbook of terms. Enactive Systems Books, 191-192.

12. Orio, N., Schnell, N., ANd Wanderley, M.M. 2001. Input Devices for Musical Expression : Borrowing Tools from HCI. New Interfaces for Musical Expression, 1518.

13. JordÀ, S., Gómez-Marín, D., Faraldo, A., and Herrera, P. 2016. Drumming with style: From user needs to a working prototype. New Interfaces for Musical Expression, Queensland Conservatorium Griffith University, 365-370. 14. Geiger, C., Reckter, H., Paschke, D., Schulz, F., and Poepel, C. 2008. Towards Participatory Design and Evaluation of Theremin-based Musical Interfaces. New Interfaces for Musical Expression. $\triangleq$

15. Tragtenberg, J.N., Calegario, F., Cabral, G., and Ramalho, G.L. 2019. Towards the Concept of Digital Dance and Music Instruments. New Interfaces for Musical Expression, UFRGS, 89-94. $\longleftarrow$

16. Nath, A. AND Young, S. 2015. VESBALL: A ball-shaped instrument for music therapy. New Interfaces for Musical Expression, Louisiana State University, 387-391.

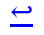

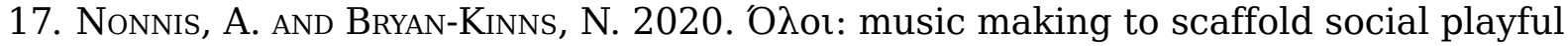
activities and self-regulation. New Interfaces for Musical Expression, Birmingham City University, 557-558. $\doteq$

18. Murray-Browne, T., Aversano, D., Garcia, S., et Al. 2014. The Cave of Sounds: An Interactive Installation Exploring How We Create Music Together. New Interfaces for Musical Expression, Goldsmiths, University of London, 307-310. 
19. BRyan-KinNs, N. AND ZIJIN, L. 2020. ReImagining: Cross-cultural Co-Creation of a Chinese Traditional Musical Instrument with Digital Technologies. New Interfaces for Musical Expression, Birmingham City University, 382-387. 20. Ko, C.L. and Oenlberg, L. 2020. Touch Responsive Augmented Violin Interface System II: Integrating Sensors into a 3D Printed Fingerboard. New Interfaces for Musical Expression, Birmingham City University, 166-171.

21. Yoshimura, F. AND KazUhiro jo. 2019. A "voice" instrument based on vocal tract models by using soft material for a 3D printer and an electrolarynx. New Interfaces for Musical Expression, UFRGS, 411-412.

22. Gallin, E. ANd Sirguy, M. 2009. Sensor Technology and the Remaking of Instruments from the Past. New Interfaces for Musical Expression, 199-202. 23. Blazey, R. 2017. Kalimbo: an Extended Thumb Piano and Minimal Control Interface. New Interfaces for Musical Expression, Aalborg University Copenhagen, 501-502.

24. The Ex. 2011. Double Order. Catch My Shoe. $\subseteq$ 25. Bau, O., TAnaka, A., And Mackay, W.E. 2008. The A20 : Musical Metaphors for Interface Design. New Interfaces for Musical Expression, 91-96. 26. Bowers, J. ANd Archer, P. 2005. Not Hyper, Not Meta, Not Cyber but InfraInstruments. New Interfaces for Musical Expression, 5-10. $\doteq$ 27. GADD, A. AND FELS, S.S. 2002. MetaMuse: Metaphors for Expressive Instruments. New Interfaces for Musical Expression, 65-70. $\uplus$ 28. Strathern, M. 2006. A community of critics? Thoughts on new knowledge. Journal of the Royal Anthropological Institute 12, 1, 191-209. (quoted) $ヒ$ 29. Jensenius, A.R. And Lyons, M.J. 2017. A NIME Reader: Fifteen Years of New Interfaces for Musical Expression. Springer. $\triangleq$ 30. Jensenius, A.R. And Lyons, M.J. 2016. Trends at NIME-Reflections on Editing" A NIME Reader". New Interfaces for Musical Expression. $\subseteq$

31. Dion, M.L., Sumner, J.L., And Mitchell, S.M. 2018. Gendered citation patterns across political science and social science methodology fields. Political Analysis 26, $3,312-327$. 
32. BorN, G. 2010. For a relational musicology: music and interdisciplinarity, beyond the practice turn. Journal of the Royal Musical Association 135, 2, 205-243. $ヒ$ 33. Marquez-Borbon, A. And Stapleton, P. 2015. Fourteen Years of NIME: The Value and Meaning of 'Community' in Interactive Music Research. New Interfaces for Musical Expression, Louisiana State University, 307-312.

34. Dobrian, C. and Koppelman, D. 2006. The E in NIME: Musical Expression with New Computer Interfaces. New Interfaces for Musical Expression. $\bullet$

35. Barbosa, J., Malloch, J., Wanderley, M., and Huot, S. 2015. What does

"Evaluation" mean for the NIME community? New Interfaces for Musical Expression, Louisiana State University. $ヒ$

36. Cantrell, J. 2017. Designing Intent: Defining Critical Meaning for NIME Practitioners. New Interfaces for Musical Expression. $\_$

37. Carey, B. And Johnston, A. 2016. Reflection On Action in NIME Research: Two Complementary Perspectives. New Interfaces for Musical Expression. $\subseteq$ 38. Birnbaum, D., Fiebrink, R., Malloch, J., and Wanderley, M.M. 2005. Towards a Dimension Space for Musical Devices. New Interfaces for Musical Expression, 192195. $\doteq$

39. Morreale, F., McPherson, A., and Wanderley, M. 2018. NiME Identity from the Performer's Perspective. New Interfaces for Musical Expression. $\triangleq$ 40. Xambo Sedo, A., Saue, S., Jensenius, A.R., Støckert, R., and Brandtsegg, Ø. 2019. NIME prototyping in teams: A participatory approach to teaching physical computing. Proceedings of the International Conference on New Interfaces for Musical Expression, 216-221.

41. JordÀ, S. ANd Mealla, S. 2014. A methodological framework for teaching, evaluating and informing NIME design with a focus on expressiveness and mapping. Proceedings of the International Conference on New Interfaces for Musical Expression, 233-238.

42. Morreale, F. and McPherson, A. 2017. Design for Longevity: Ongoing Use of Instruments from NIME 2010-14. New Interfaces for Musical Expression. $\subseteq$ 
43. McPherson, A., Berdahl, E., Jensenius, A.R., Lyons, M.J., Bukvic, I.I., and Knudsen, A. 2016. NIMEhub: Toward a repository for sharing and archiving instrument designs. .

44. BiN, S.M.A. 2018. The Show Must Go Wrong: Towards an understanding of audience perception of error in digital musical instrument performance. .

45. Keyes, O., Hoy, J., and Drouhard, M. 2019. Human-computer insurrection: Notes on an anarchist HCI. Proceedings of the 2019 CHI Conference on Human Factors in Computing Systems, 1-13. $\subseteq$

46. Light, A. 2011. HCI as heterodoxy: Technologies of identity and the queering of interaction with computers Interacting with computers 23, 5, 430-438.

47. Spiel, K., Gerling, K., Bennett, C.L., et Al. 2020. Nothing about us without us: Investigating the role of critical disability studies in hci. Extended Abstracts of the 2020 CHI Conference on Human Factors in Computing Systems, 1-8.

48. Bardzell, S. 2010. Feminist HCI: taking stock and outlining an agenda for design. Proceedings of the SIGCHI conference on human factors in computing systems, 1301-1310.

49. Jaroszewski, S., LotTridge, D., Haimson, O.L., And Quehl, K. 2018. “ Genderfluid” or" Attack Helicopter" Responsible HCI Research Practice with Non-binary Gender Variation in Online Communities. Proceedings of the 2018 CHI Conference on Human Factors in Computing Systems, 1-15.

50. Linehan, C. AND KiRMAn, B. 2014. Never mind the bollocks, i wanna be anarCHI: a manifesto for punk HCI. In: CHI'14 Extended Abstracts on Human Factors in Computing Systems. 741-748. $\_$

51. Haslhofer, B., IsaAc, A., And Simon, R. 2018. Knowledge graphs in the libraries and digital humanities domain. arXiv preprint arXiv:1803.03198.

52. Drakopoulos, G., Spyrou, E., Voutos, Y., and Mylonas, P. 2019. A semantically annotated JSON metadata structure for open linked cultural data in Neo4j. Proceedings of the 23rd Pan-Hellenic Conference on Informatics, 81-88.

53. Sснӧсн, C. 2013. Big? smart? clean? messy? Data in the humanities. Journal of digital humanities 2, 3, 2-13. 